\title{
DOPPLER COLOR EN EL ESTUDIO DE LA MAMA: ¿CÓMO LO HACEMOS NOSOTROS?
}

Drs. Eleonora Horvath(1), María Julia Cuitiño ${ }^{(2)}$, Miguel Ángel Pinochet $T^{(1)}$ Patricia Sanhueza ${ }^{(3)}$.

1. Radiólogos, Clínica Alemana de Santiago, Facultad de Medicina Universidad del Desarrollo. Chile.

2. Medica residente de Diagnóstico por Imágenes, Hospital Privado de Comunidad. Mar del Plata, Argentina.

3. Radióloga, Hospital de la Serena y Coquimbo. Chile.

\section{COLOR DOPPLER IN THE STUDY OF THE BREAST: HOW DO WE PERFORM IT?}

\begin{abstract}
The contribution of color Doppler sonography in the study of breast cancer remains a topic of discussion. However, in the daily clinical practice it has become an indispensable instrument, and an integral part of the breast ultrasound (US). The aim of this paper is to demonstrate its utility based on the available evidence as well as on our experience. We describe the technical considerations necessary to conduct a good study, the Doppler signs of benignity/malignancy in focal lesions of the breast and the benefits of its routine use in day-to-day practice. In our experience, it is a useful tool for this purpose. Neverthless, its diagnostic impact as described in the literature is variable. To evaluate its real usefulness, prospective studies along with standardization of the evaluation technique would be required.
\end{abstract}

Key words: Angiogenesis, Breast Neoplasms, Breast Ultrasound, Doppler sonography, Vascularity.

Resumen: La contribución del Doppler color en el estudio de la mama sigue siendo un tema en discusión. No obstante, en la práctica clínica diaria se ha convertido en un instrumento indispensable, formando parte integral del Ultrasonido (US) mamario.

El objetivo de este artículo es demostrar su utilidad según la evidencia disponible y a través de nuestra experiencia.

Se describen las consideraciones técnicas indispensables para realizar un buen estudio, los signos Doppler de benignidad / malignidad en lesiones focales de la mama y las ventajas de su uso rutinario en la práctica diaria.

En nuestra experiencia es una herramienta útil para este propósito, sin embargo su impacto diagnóstico descrito en la literatura es variable. Para evaluar su real utilidad, se requiere de la realización de estudios prospectivos y de la estandarización la técnica.

Palabras clave: Angiogénesis, Ecografía Doppler, Neoplasia mamaria, Ultrasonido mamario, Vascularización.

Horvath E. Doppler color en el estudio de la mama: ¿Cómo lo hacemos nosotros? Rev Chil Radiol 2011; 17(1): $19-27$. Correspondencia: Dra. Eleonora Horvath / eleonora.horvath@gmail.com

Trabajo recibido el 07 de septiembre de 2010, aceptado para publicación el 02 de febrero de 2011.

\section{Introducción}

El rol del Doppler color en la patología mamaria sigue siendo un tema controversial ${ }^{(1-3)}$. En este tópico no se dispone de evidencia sólida debido a que no se han realizado trabajos en grandes poblaciones, de manera que no existen estudios prospectivos randomizados y controlados. Los resultados -obtenidos en la mayoría de pequeñas series- son heterogéneos, con conclusiones no siempre confiables. Al no existir estándares bien definidos, la evaluación de la vascularización de las lesiones mamarias aún no es obligatoria en el léxico BI-RADS US ${ }^{(4-7)}$.

Pese a lo anterior, con los avances tecnológicos en la industria de los transductores y con la experiencia adquirida en ultrasonido (US) mamario, la técnica Doppler se utiliza hoy cada vez con mayor convencimiento. 
El propósito de este artículo es analizar el uso del Doppler color en la patología mamaria a la luz de la evidencia disponible y describir cómo puede mejorar el rendimiento del US modo $B$ en el examen rutinario de la mama, según nuestra experiencia.

\section{Consideraciones técnicas}

Es necesario utilizar transductores de alta frecuencia (idealmente de 12 a $18 \mathrm{MHz}$ ) para ser capaz de evaluar señal en vasos menores a 0,1 mm de diámetro con flujo de baja velocidad. Se emplean diferentes modalidades Doppler para el estudio mamario, tales como: Doppler power, modo espectral, Doppler color con o sin inyección de medio de contraste.

El Doppler color es una herramienta de fácil uso, disponible en la mayoría de los ecógrafos en la actualidad. Proporciona información sobre presencia de vascularización en los tejidos, permite precisar la dirección del flujo y distinguir una vena de una arteria de acuerdo al tipo de curva espectral observada.

Con el fin de demostrar flujos muy lentos, es necesario trabajar con una mínima presión sobre la mama, evitando así la compresión de los vasos. Además se necesita ajustar una serie de parámetros que permiten obtener óptima coloración de los vasos [PRF entre $700-1000 \mathrm{~Hz}$, filtro de pared lo más bajo posible (50 a $100 \mathrm{~Hz}$ max.), algoritmo adecuado para eliminar artefactos de movimiento, máxima ganancia (85-90\%), persistencia media, caja sin angulación].

El Doppler power es óptimo para detectar pequeños vasos con flujo lento, ya que es independiente del ángulo Doppler, lo que representa una gran ventaja en la evaluación de las lesiones mamarias. Se estima que posee 2 a 5 veces mayor sensibilidad que el Doppler color ${ }^{(3,5)}$, no obstante esta afirmación no se ha confirmado en nuestra práctica. Sus desventajas son: mayor número de artefactos y falta de información acerca de la velocidad y dirección del flujo, por lo que no permite diferenciar arterias de venas ${ }^{(3,5)}$.

La medición de parámetros de flujo en Doppler espectral teóricamente podría ayudar en la caracterización de los nódulos mamarios; se ha demostrado una alta velocidad y pulsatilidad con un peak sistólico mayor en las lesiones malignas que en las benignas $^{(5,8-15)}$. Sin embargo, los resultados dependen de otros factores (por ejemplo de la histología de la lesión) y en la práctica estas mediciones resultan difícilmente reproducibles porque son operador y equipo dependientes, existiendo una gran variabilidad inter-observador

Como línea de investigación, se intenta demostrar la microcirculación de los nódulos mamarios al aumentar la sensibilidad del Doppler color con la inyección de medio de contraste ${ }^{(9)}$. No obstante, incluso con la inyección de microburbujas se mantiene la superposición entre lesiones benignas hipervascularizadas y malignas hipovasculares, por lo que no se ha masificado su uso.

Por su simplicidad, el Doppler color y el Doppler power han logrado mayor popularidad en el trabajo cotidiano.

\section{Potencialidades del Doppler color para diferenciar nódulo benigno/maligno}

Mientras las lesiones mamarias benignas poseen vasos nativos maduros de arquitectura armoniosa, los tumores malignos secretan factores angiogénicos que estimulan el crecimiento de los vasos anómalos ${ }^{(16)}$. Se cree que la neoangiogénesis es causada por la proteína angiogenina que genera una red vascular local altamente densa en capilares, loops y shunts arteriovenosos. Los neovasos son tortuosos, de calibre irregular y de paredes finas, sin músculo liso(17).

En resonancia magnética $(R M)$ mamaria dinámica se busca demostrar la neovascularización tumoral con la inyección de gadolinio, puesto que estos vasos anómalos se impregnan precozmente con el contraste. En Doppler color no es posible identificar los capilares, pero sí los vasos aferentes e intra-tumorales de mayor calibre. Por lo anterior, se podría pensar que una vascularización aumentada en Doppler color incrementa la posibilidad de malignidad de un nódulo. Sin embargo, la hipervascularización en una lesión mamaria no es por sí misma un adecuado predictor de malignidad, existiendo una gran superposición entre la irrigación de las lesiones benignas y malignas ${ }^{(9,18)}$. Miltz ilustra bien lo anterior en su serie de 400 nódulos biopsiados: el $45 \%$ de las lesiones benignas, el $80 \%$ de las malignas invasoras y el $25 \%$ de las malignas in situ presentaron flujo ${ }^{(19)}$. Al considerar sólo el "carácter vascularizado o no" de un nódulo como criterio de malignidad, los resultados muestran baja sensibilidad, especificidad y valores predictivos ${ }^{(1)}$. Queda así en evidencia que el aumento de vascularización al Doppler color como factor único no puede establecer el diagnóstico de malignidad y que el aporte de esta técnica se debe enfocar de otra manera: hoy se lo propone como un elemento adicional, complementario al US bidimensional para analizar los vasos aferentes y la vascularización interna de los nódulos mamarios.

En varios trabajos ${ }^{(1,3,8-12,15,18,20-22)}$ se han establecido criterios semiológicos para lesiones benignas y malignas, según localización y morfología de los vasos detectados.

\section{Signos Doppler de benignidad}

Los nódulos benignos, clasificados como categoría BI-RADS 3, presentan signos ecográficos bien definidos en modo $\mathrm{B}^{(6)}$ : son lesiones de forma oval, con hasta 2-3 lobulaciones suaves, de contornos circunscritos y con orientación paralela a la piel; la hiperecogenicidad es otro elemento de benignidad. Adicionalmente, en este tipo de lesiones mamarias, los siguientes signos Doppler indican benignidad(22,23): 
- Vasos capsulares de trayecto recto o curvilíneo, de calibre regular y de distribución armoniosa (Figura 1). Es un hallazgo frecuente en las lesiones benignas, tales como fibroadenomas ${ }^{(18)}$.

- El hallazgo de "arteria y vena juntas" (Figura 2) según nuestra experiencia constituye otro signo de benignidad. Una arteria acompañada por su vena en la periferia o espesor de un nódulo obedece a una condición anatómica normal. Estos vasos nativos son diferentes de los neo-vasos tortuosos, de distribución anárquica, producto de la angiogénesis tumoral. En nuestra serie, aún en revisión, el $98,5 \%$ de los nódulos que presentaron este hallazgo resultaron benignos (140/142). Se trata generalmente de fibroadenomas, pero también se observa en otras lesiones benignas, tales como papilomas, adenoma lactante, ganglios intramamarios, etc.
- El carácter avascular de una lesión de aspecto morfológico benigno ${ }^{(18)}$, de categoría BI-RADS US 3 sin señal color, corresponde frecuentemente a un quiste con contenido espeso o a un nódulo sólido benigno, tipo fibroadenoma, sin capacidad de neoangiogénesis (Figura 3a); en nuestra experiencia este signo significa benignidad en la gran mayoría de los casos. Por otra parte, cabe destacar que con la tecnología actual en nódulos de morfología dudosa sospechosa (de categoría BI-RADS 4b, 4c y 5) no siempre somos capaces de visualizar los vasos intra-lesionales, solo percibimos los neovasos aferentes tortuosos, característicos de malignidad (Figura 3b). En este contexto, la aparente "avascularidad" no debe interpretarse como signo de benignidad.

- En cuanto al número de los vasos aferentes, las lesiones benignas tienden a tener un polo vascular periférico único ${ }^{(18)}$.

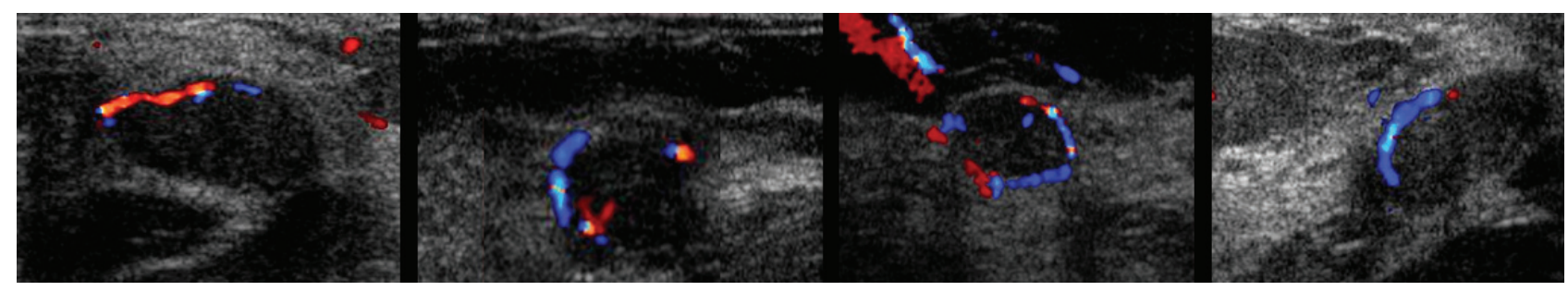

Figura 1. La presencia de vasos capsulares de calibre regular, de trayecto recto-curvilíneo y de distribución armoniosa es un hallazgo frecuente en las lesiones benignas, tales como fibroadenomas.

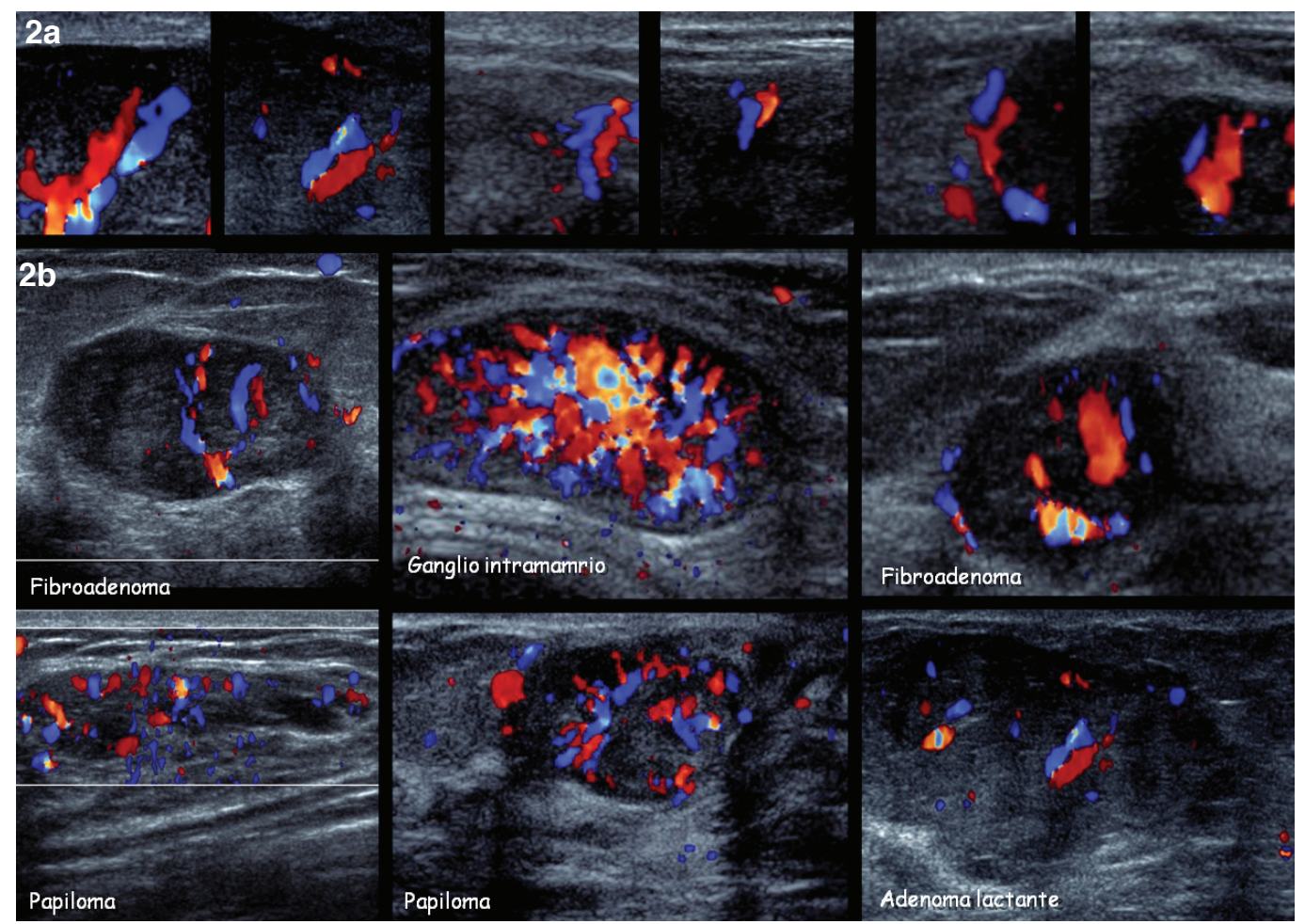

Figura 2. Signo de "arteria y vena juntas": una arteria acompañada por su vena en la periferia o espesor de un nódulo, señalando una condición anatómica normal (a). Poco frecuente $(12 \%$ en nuestras biopsias core), pero de alto valor predictivo para benignidad; se observa en fibroadenomas, papilomas, ganglios intramamarios, adenomas lactantes, entre otros (b). 

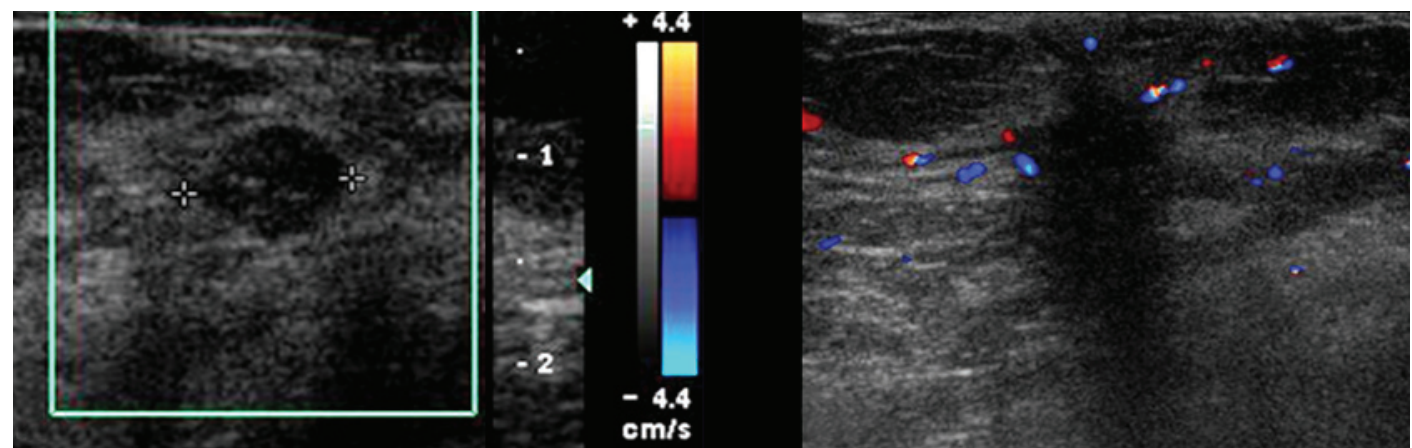

Figura 3. La avascularidad de una lesión mamaria sólo representa signo de benignidad en nódulos de aspecto morfológico benigno/probablemente benigno, tipo BI-RADS US 3 ó 4A, por ejemplo en casos de fibroadenomas y quistes con contenido espeso (a). En algunos nódulos sospechosos (BI-RADS US 5), con la tecnología actual no se logra demostrar vasos intralesionares (b). En esta situación el aparente "avascularidad" no se debe interpretar como signo de benignidad.

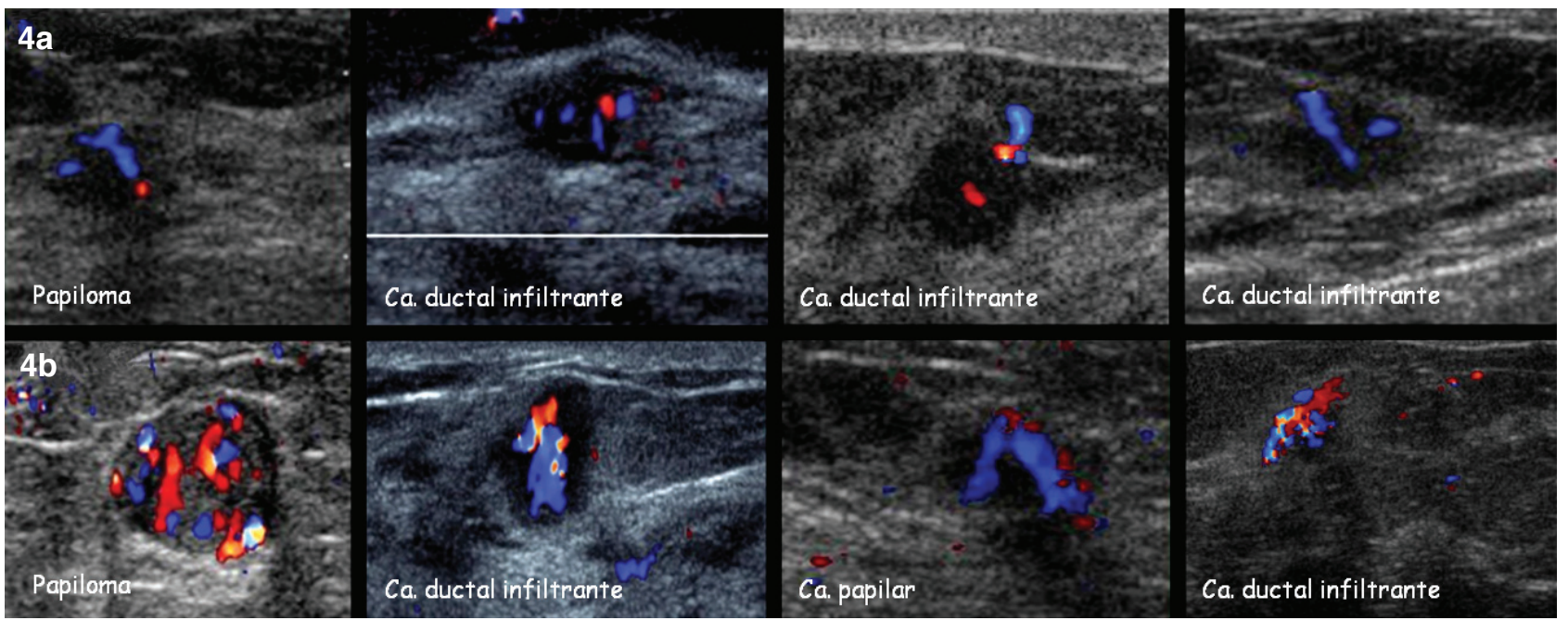

Figura 4. La existencia de vasos intra-nodulares sin vasos capsulares aumenta el grado de sospecha en nódulos indeterminados o sospechosos, de categoría BI-RADS US 4 y 5 (a), especialmente cuando gran \% de la imagen del nódulo se encuentra vascularizado (b). Generalmente se trata de papilomas o de lesiones malignas.

\section{Signos Doppler de malignidad}

Los nódulos catalogados como indeterminados o sospechosos en modo B (BI-RADS 4 y 5 ) tienen forma y contornos irregulares, con espículas o microlobulaciones, son más altos que anchos, contienen frecuentemente microcalcificaciones y pueden generar sombra acústica posterior o un halo hiperecogénico por reacción desmoplastica ${ }^{(24)}$. En este tipo de lesiones, los siguientes elementos adicionales sugieren malignidad al Doppler color:

- Presencia de vasos centrales en ausencia de vasos capsulares ${ }^{(25)}$ (Figura 4a). Cuando gran porcentaje de la imagen del nódulo se cubre con píxeles de color (Figura 4b), habitualmente se trata de una lesión maligna o papilomatosa.
- Las arterias aferentes o intra-nodulares tortuosas, de distribución anárquica y calibre variable por ramificaciones irregulares, corresponden a los típicos neovasos, producto de la angiogénesis tumoral(25) (Figura 5).

- Los vasos aferentes que penetran perpendicularmente al nódulo, sin pasar por vasos capsulares, representan un hallazgo altamente sugerente de malignidad $^{(2,18,25)}$ (Figura 6).

- El gran número de pedículos vasculares aferentes, junto a la alta densidad vascular intra-nodular, según varios autores se correlaciona con la agresividad tumoral y con el riesgo metastático(2) (Figura 7 y 3b). Santamaría ${ }^{(26)}$ encontró una relación lineal entre el tamaño tumoral, número de vasos 


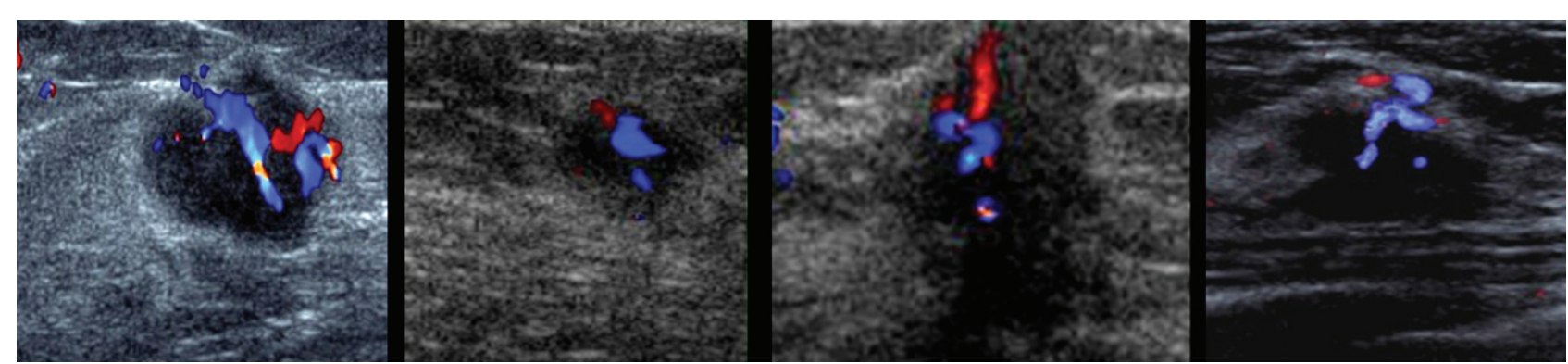

Figura 5. Los neovasos intra-lesionares tortuosos, de distribución anárquica y calibre variable por ramificaciones irregulares, aumentan categóricamente el BI-RADS US de un nódulo mamario.

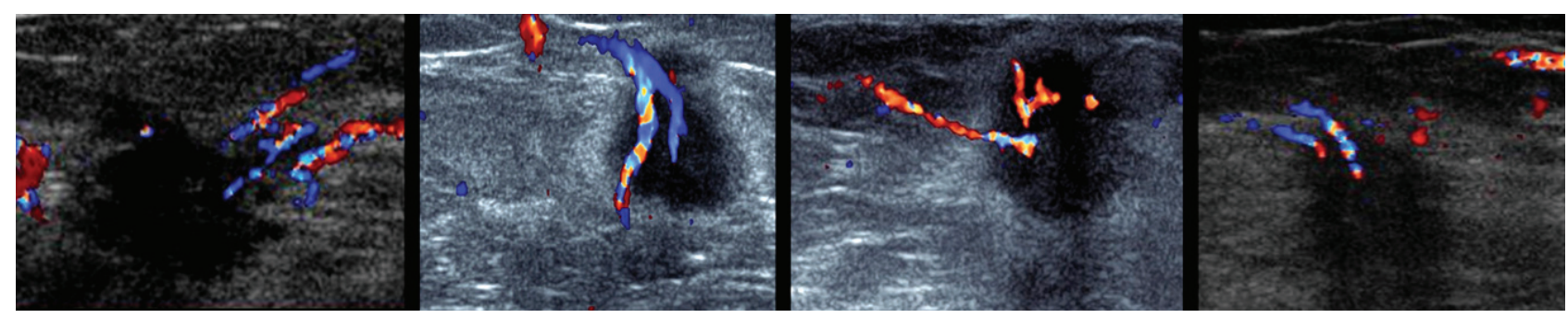

Figura 6. Los vasos aferentes penetrantes que no cursan por vasos capsulares sino directamente al nódulo, representan un hallazgo altamente sugerente de malignidad.

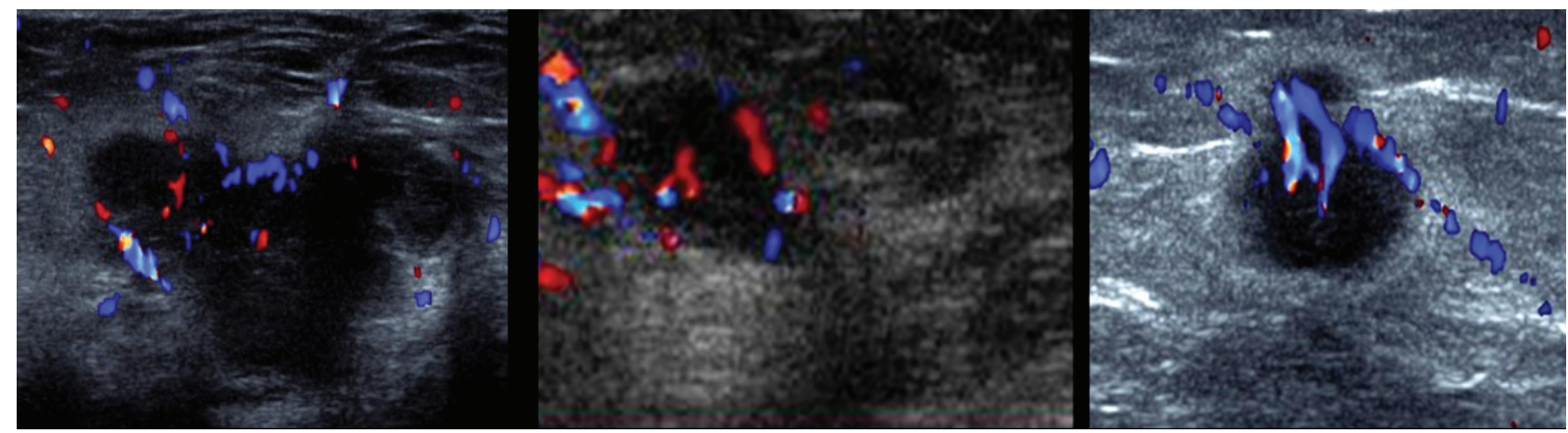

Figura 7. El gran número de pedículos vasculares aferentes, al parecer se correlaciona con la agresividad tumoral y con el riesgo metastático.

aferentes y la existencia de metástasis axilares. Según la teoría de Byers ${ }^{(27)}$, uno de los factores de la diseminación tumoral es el movimiento de células o de embolias tumorales desde la masa tumoral hacia los vasos linfáticos o venas que presentan una circulación laminar. Esta teoría sugiere que mientras mayor es la vascularización arterial, mayor efecto de succión va a ejercer el flujo laminar, favoreciendo así el desprendimiento de las células tumorales.

Utilizando estos criterios de sospecha, el Doppler color posee una sensibilidad variable para cáncer, evaluada en 68-99\% y una especificidad de $43-97 \%$, VPP de 58 a $88 \%$ y VPN de 80 a $100 \%$ según diferentes estudios ${ }^{(2,3,5,18,23,28)}$. Esta gran inconsistencia en el resultado de un estudio al otro se explica por las diferencias en las técnicas utilizadas (Doppler color, Power angio o inyección de contraste).

Si bien en general el registro de los parámetros de Doppler pulsado no ofrece elementos específicos para discriminar benigno de maligno, según del Cura ${ }^{(1)}$, la malignidad del nódulo es altamente probable, cuando el diástole desaparece o está invertido. 


\section{Ventajas del uso rutinario del Doppler color}

El Doppler color se convirtió en una herramienta indispensable y actualmente forma parte integral de la US mamaria.

Con la tecnología adecuada, los hallazgos Doppler antes mencionados permiten una mejor caracterización de los nódulos BI-RADS 3 y 4, cuyo porcentaje de riesgo para cáncer es muy variable (inferior al $2 \%$ y 3 a $94 \%$, respectivamente) ${ }^{(6)}$. Aunque no se cuenta con evidencia sólida, solo con opinión de expertos ${ }^{(2,6)}$ que compartimos esta técnica, permite una mejor selección de las lesiones que requieren estudio histológico en el trabajo cotidiano. Si aceptamos la hipótesis que la presencia de los signos "Doppler benignos" refuerzan el aspecto benigno de los nódulos, es posible "bajar" el BI-RADS de un nódulo probablemente benigno/ indeterminado en modo B y de esta manera preconizar su seguimiento por imágenes (ej. en nódulos BI-RADS 3 y 4 A) (Figura 8a).

Al contrario, el "color-patrón maligno" característico aumenta el nivel de sospecha de las lesiones clasificadas previamente como probablemente benignas/indeterminadas, argumentando a favor de la necesidad de verificación histológica(2,6) (Figura 8b).

Considerando lo anterior, si logramos discriminar mejor entre nódulos probablemente benignos e indeterminados, podríamos disminuir las biopsias innecesarias. En el estudio clásico de Stavros ${ }^{(29)}$, el índice de biopsia con resultado benigno versus no benigno (= maligno y lesiones de alto riesgo) es de
5 a 1. En la serie de Buchberger ${ }^{(30)}$, esta relación es aún más alta, de 6.3 a 1 En nuestra práctica actual (364 biopsias Core en el año 2010) este índice es de 1.7 a 1, resultado que pensamos que en gran parte se debe al uso rutinario del Doppler color.

Además de la caracterización de los nódulos, el Doppler color ayuda en diversas circunstancias, como por ejemplo:

- Diferenciar un lobulillo de grasa de un nódulo sólido (Figura 9a); mientras el primero es avascular, en el nódulo real existe la posibilidad de demostrar vasos (siempre y cuando éstos sean detectables al Doppler color).

- Reconocer un ganglio intramamario (Figura 9b), en base a a su vascularización hiliar.

- Las adenopatías metastásicas se so spechan al Doppler color, debido al desarrollo de vasos capsulares, que es un signo casi patognomónico de compromiso tumoral metástasico (Figura 9c).

- Diferenciar un tumor vascularizado de un quiste con contenido espeso (Figura 9d), debido a su carácter avascular.

- Identificar tumores intra-quísticos e intraductales de tipo papiloma (Figura 10), gracias a la vascularización del tumor. Se trata de lesiones muy irrigadas, en las que no es posible diferenciar entre variante benigna y maligna en US. Excepcionalmente, lesiones más antiguas pueden aparecer poco o no vascularizados a causa de infartos ${ }^{(2,7)}$.

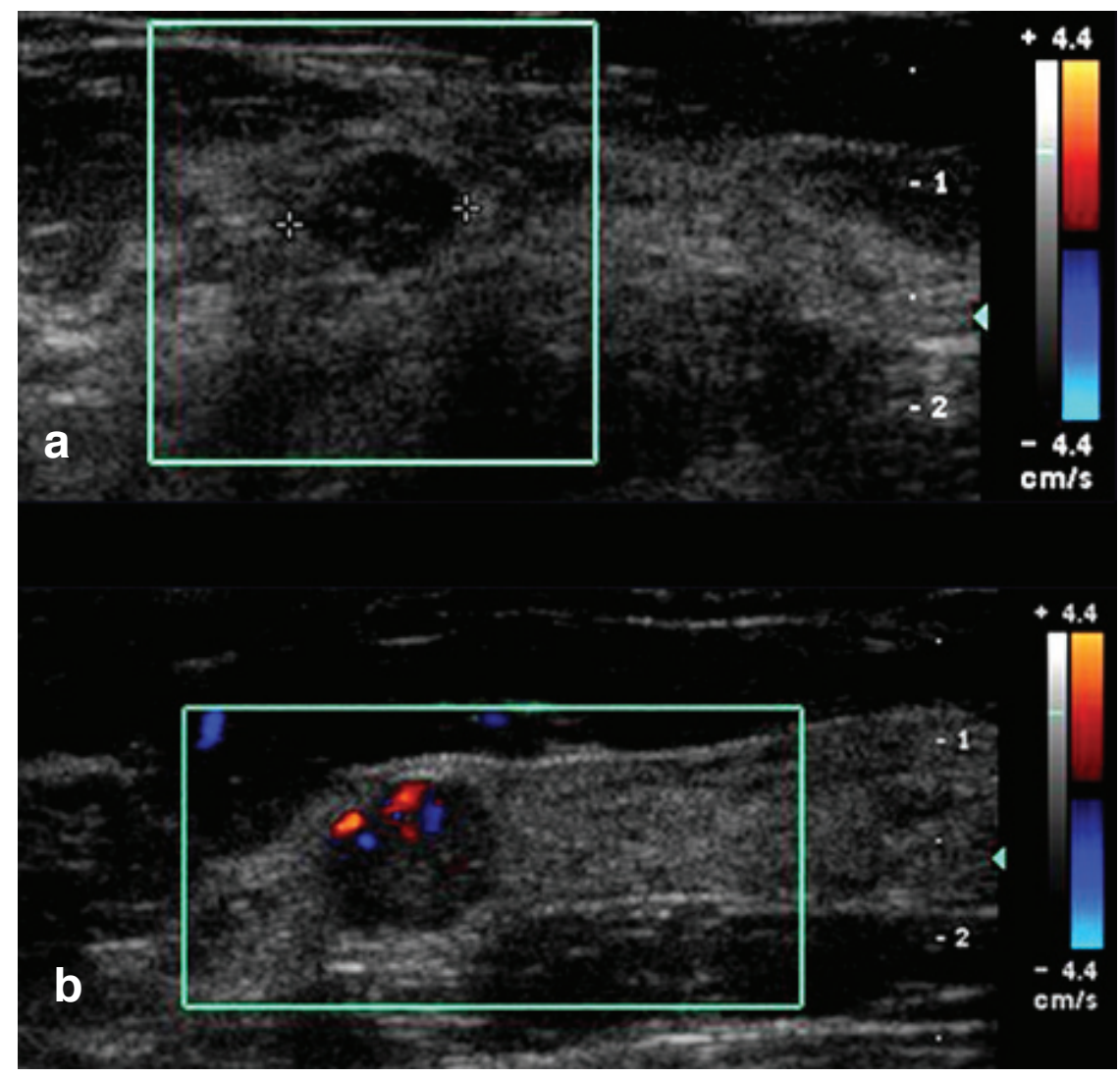

24
Figura 8. El hallazgo de un nódulo indeterminado con signo Doppler benigno (a), por ejemplo la avascularidad, permite recomendar un seguimiento ecográfico en vez de biopsia. Un signo Doppler sospechoso (ej. numerosos vasos intra-nodales sin vasos capsulares) en un nódulo caracterizado como indeterminado en escala de grises (b) aumenta la sospecha y por ende, se recomienda indicar biopsia. 


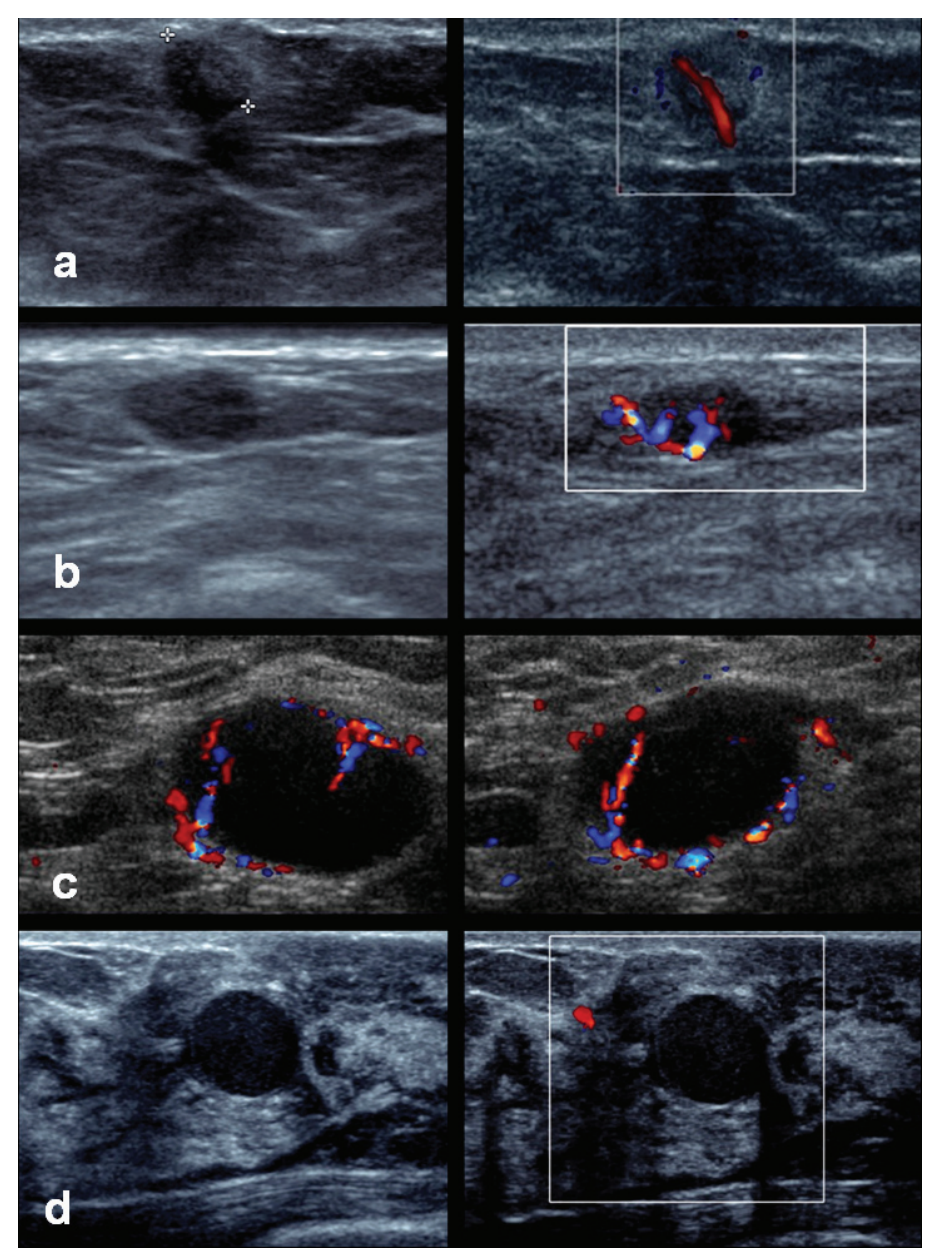

Figura 9. Algunas aplicaciones del Doppler color: Diferenciar un lobulillo de grasa de un nódulo sólido (a): mientras el primero es habitualmente avascular, en el nódulo real eventualmente se puede demostrar vasos. Reconocer un ganglio intramamario (b) gracias a su vascularización hiliar. Las adenopatías metastásicas son enteramente hipoecogénicas y desarrollan vasos capsulares (c), signo casi patognomónico de compromiso metastásico. Diferenciar un quiste con contenido espeso $(d)$ de un tumor vascularizado gracias a la avascularidad del contenido quístico.

Figura 10. El Doppler color ayuda a identificar tumores intra-quísticos e intra-ductales de tipo papiloma, gracias a la vascularización del tumor $(a, b, c)$. Incluso en tumores pequeños se puede visualizar el tallo vascular con Doppler color (b). Es excepcional que un papiloma aparezca pobremente vascularizado (c) o avascular; esto ocurre por infarto en lesiones más antiguas.

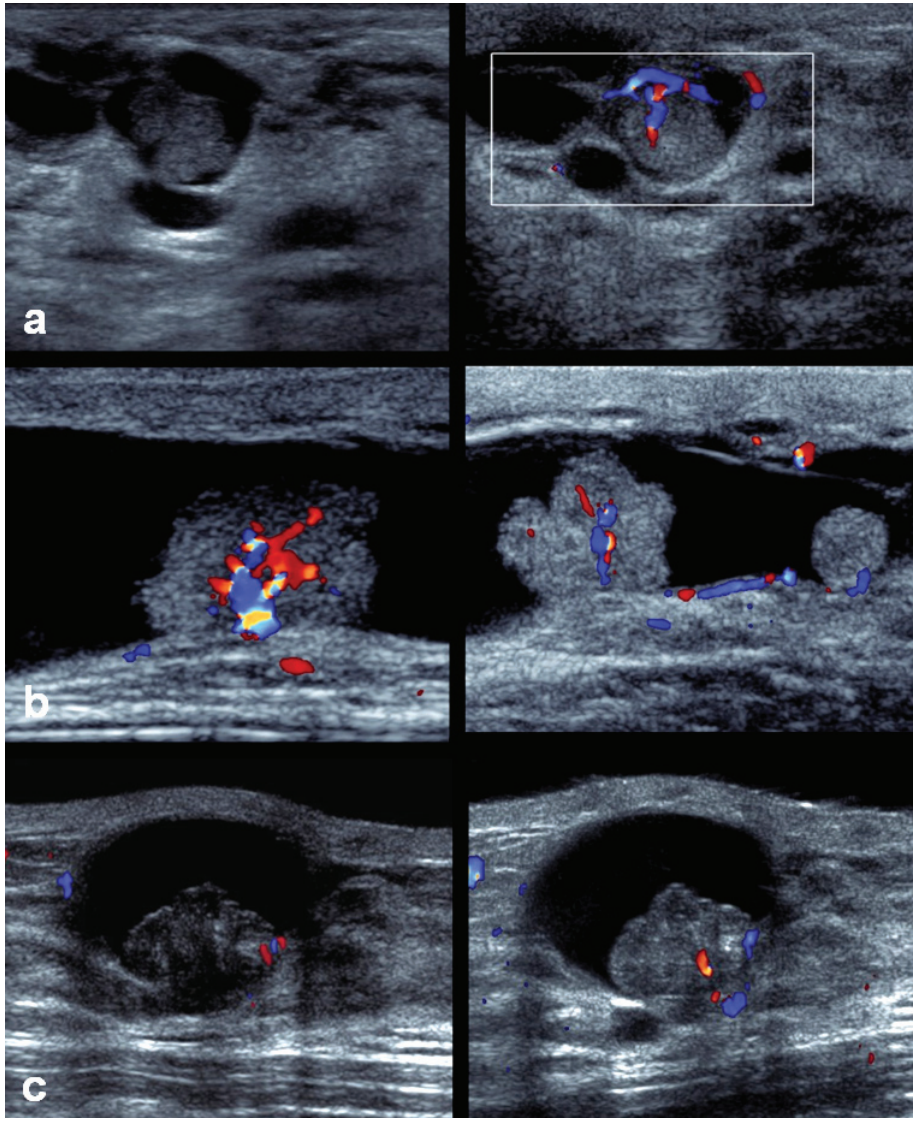




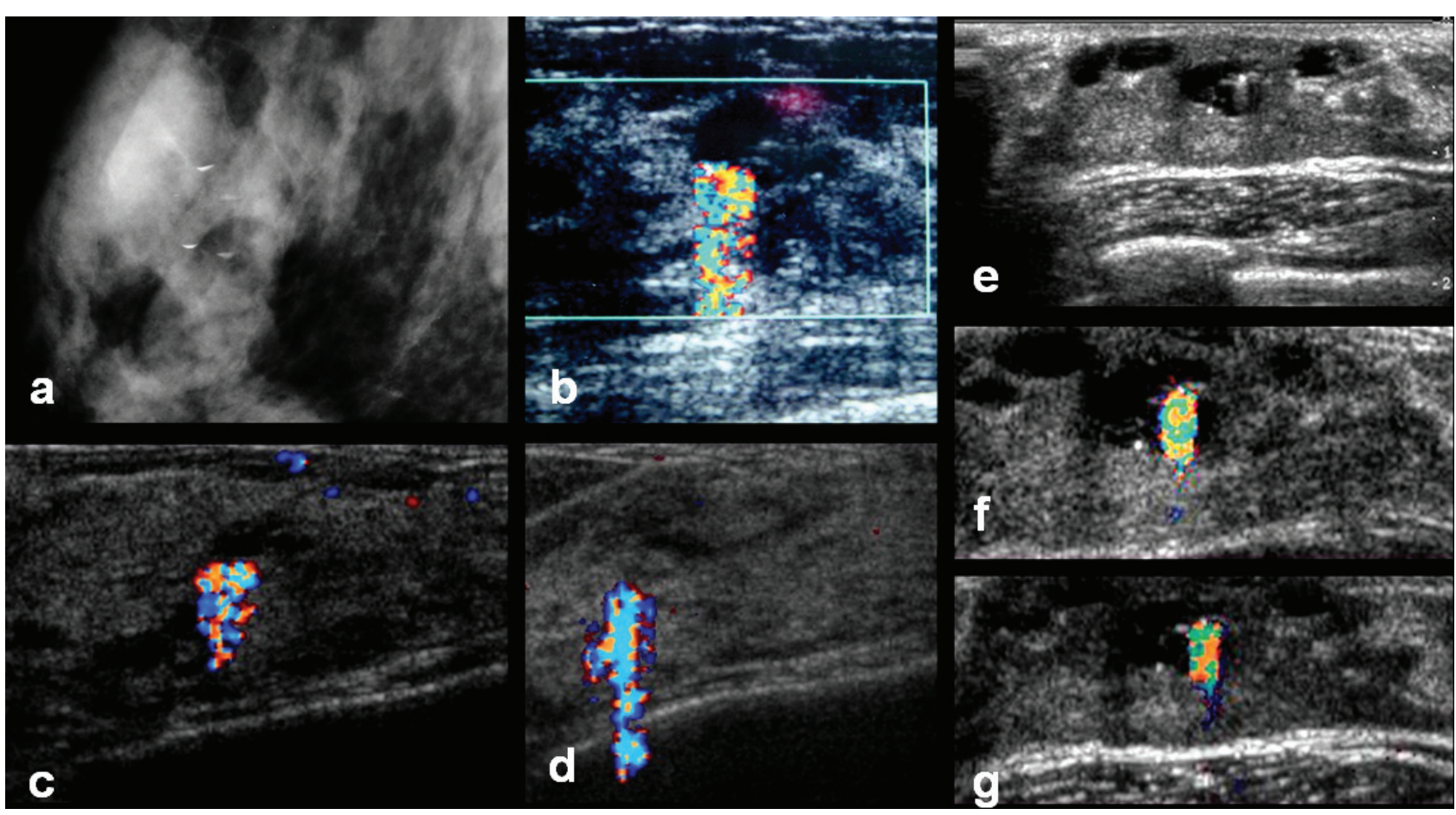

Figura 11. Las microcalcificaciones de tipo "lechada de cal" detectadas en mamografía (a) pueden ser identificadas en US gracias al artefacto de centelleo que provocan al estudiarlas con Doppler color (b-d). En aproximadamente el $45 \%$ de los quistes retroareolares en adolescentes se encuentra este tipo de calcificación (e) que produce intenso centelleo ( $f$ - $g$ ).

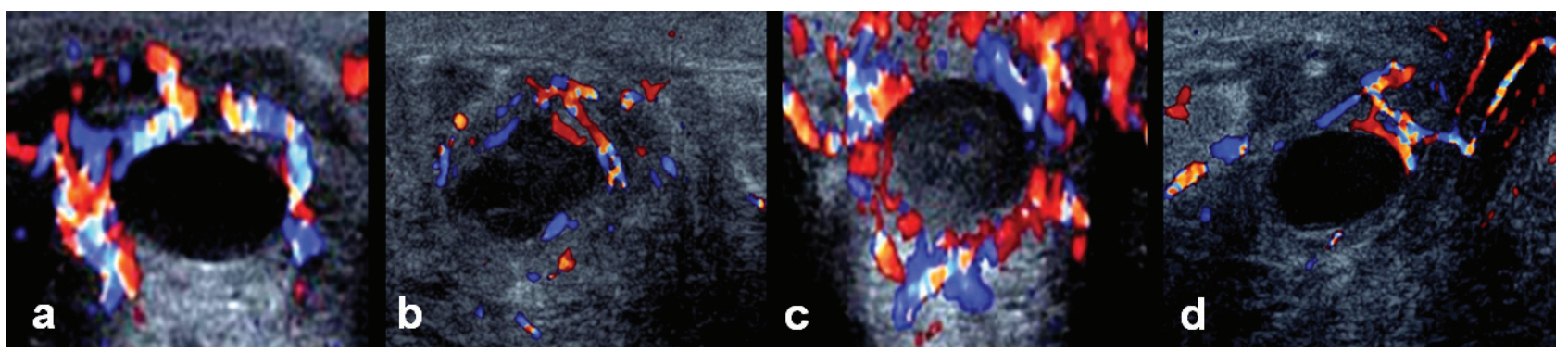

Figura 12. Un proceso inflamatorio agudo en la mama -como ocurre por ejemplo en casos de los quistes retroareolares sobreinfectados en la adolescencia- causa una gran hiperemia peri-lesional en la vecindad, visible al Doppler color (a-e).

- Microcalcificaciones del tipo "lechada de cal", localizadas en microquistes o conductos se pueden identificar como tales al Doppler color visualizando artefacto de centelleo (Figura 11).

- El proceso inflamatorio agudo peri-ductal o periquístico se demuestra por la hipervascularización de estas estructuras. La gran hiperemia parietal y peri-lesional es característica también en abscesos mamarios y quistes retroareolares sobreinfectados (Figura 12).

- La evaluación de la lesión con Doppler color antes de efectuar biopsias bajo US, es de gran ayuda. En presencia de numerosos vasos o arterias de gran calibre, se aconseja usar vasoconstrictores junto con la anestesia local (ej. epinefrina), para disminuir la posibilidad de hemorragia.

\section{Recomendaciones}

EI ACR, en su primera publicación sobre el BI-RADS ecográfico ${ }^{(4)}$ incluyó la evaluación de la vascularización de lesiones mamarias y de su vecindad, sin declarar el uso obligatorio del Doppler color.

En nuestro último Consenso Nacional del 2009 se recomendó su uso rutinario, confirmando el sentido común de que el Doppler color es una herramienta que hoy debe formar parte integral de las US mamarias para optimizar el estudio.

\section{Conclusiones}

Utilizando un equipamiento adecuado, el Doppler color permite una mejor caracterización de los nódulos mamarios mediante lo cual podría disminuir el número de biopsias innecesarias. 
Su impacto diagnóstico es variable según los estudios disponibles, lo que hace necesario estandarizar la técnica de evaluación y los parámetros a considerar.

Faltan aún estudios que entreguen mayor evidencia de su real aporte; mientras tanto, su uso es recomendado.

\section{Bibliografía}

1. Del Cura JL, Elizagaray E, Zabala R, Legorburu A. Grande $D$. The use of unenhanced Doppler sonography in the evaluation of solid breast lesions. AJR Am J Roentgenol 2005; 184: 1788-1794.

2. Kwak JY, Kim EK, Kim MJ, Choi SH, Son E, Oh KK. Power Doppler sonography: evaluation of solid breast lesions and correlation with lymph node metastasis, Clinical Imaging 2008; 32: 167-171.

3. Gokalp G, Topal U, Kizilkaya E. Power Doppler sonography: Anything to add to BI-RADS US in solid breast masses? European Journal of Radiology 2009; 70: 77-85.

4. American College of Radiology (ACR) Breast Imaging Reporting and Data System Atlas (BI-RADS ${ }^{\circledR}$ Atlas). Reston, Va: @ American College of Radiology; 2003.

5. Yang WT, Tse GM, Lam PK, Metreweli C, Chang J. Correlation between color power Doppler sonographic measurement of breast tumor vasculature and immunohistochemical analysis of microvessel density for the cantitation of angiogenesis. J Ultrasound Med. 2002; 21: 1227-1235.

6. Mendelson EB, Berg WA, Merritt CR. Toward a standardized breast ultrasound lexicon, BI-RADS: ultrasound Semin Roentgenol 2001; 36: 217-225.

7. Lazarus E, Mainiero MB, Schepps B, Koelliker SL, Livingston LS. BIRADS lexicon for US and mammography: interobserver variability and positive predictive value. Radiology 2006; 239: 385-391.

8. Kim EK, Ko KH, Oh KK, Kwak JY, You JK, Kim MJ, et al. Clinical Application of the BI-RADS Final Assessment to Breast Sonography in Conjunction with Mammography. AJR 2008; 190: 1209-1215.

9. Schroeder RJ, Bostanjoglo M, Rademaker J, Maeurer J, Felix R. Role of power Doppler techniques and ultrasound contrast enhancement in the differential diagnosis of focal breast lesions. Eur Radiol 2003; 13: 68-79.

10. Sahin-Akyar G, Sumer H. Color Doppler ultrasound and spectral analysis of tumor vessels in the differential diagnosis of solid breast masses. Invest Radiol 1996; 31: $72-79$

11. Buadu LD, Murakami J, Murayama S, et al. Color Doppler sonography of breast masses: a multiparameter analysis. Clin Radiol 1997; 52: 917-923.

12. Mehta TS, Raza S, Baum JK. Use of Doppler ultrasound in the evaluation of breast carcinoma. Semin Ultrasound CT MR 2000; 21: 297-307.

13. Lee WJ, Chu JS, Huang CS, Chang MF, Chang KJ, Chen KM. Breast cancer vascularity: color Doppler sonography and histopathology study. Breast Cancer Res Treat 1996; 37: 291-298.

14. Shaheen R, Sohail S, Siddiqui KJ. Neovascularity patterns in breast carcinoma: correlation of Doppler ultrasound features with sonographic tumour morphology. JColl Physicians Surg Pak 2010; 20(3): 162-166.
15. Birdwell RL, Ikeda DM, Jeffrey SS, Jeffrey RB. Preliminary experience with power Doppler imaging of solid breast masses. AJR Am J Roentgenol 1997; 169: 703-707.

16. Madjar H, Prompeler HJ, SauerbreiW,Wolfarth R, Pfleiderer A. Color Doppler flow criteria of breast lesions. Ultrasound Med Biol 1994; 20: 849-858.

17. Weidner N, Semple JP, Welch WR, Folkman J. Tumor angiogenesis and metastasis-correlation in invasive breast carcinoma. N Engl J Med 1991; 324: 1-8.

18. Yang W, Dempsey PJ. Diagnostic Breast Ultrasound: Current Status and Future Directions. Radiol Clin N Am 2007; 45: 845-861.

19. Miltz P, Lienemann A, Kessler M, Reiser M. Evaluation of breast lesions by power doppler sonography. Eur radiol. 2001; 11: 547-554.

20. Svensson WE, Pandian AJ, Hashimoto $H$. The Use of Breast Ultrasound Color Doppler Vascular Pattern Morphology Improves Diagnostic Sensitivity with Minimal Change in Specificity. Ultraschall Med. 2010; 21.

21. Dixon JM, Walsh J, Paterson D, Chetty U. Colour Doppler ultrasonography studies of benign and malignant breast lesions. Br J Surg 1992; 79: 259-260.

22. McNicholas MM, Mercer PM, Miller JC, McDermott EW, O'Higgins NJ, MacErlean DP. Color Doppler sonography in the evaluation of palpable breast masses. AJR Am J Roentgenol 1993; 161: 765-771.

23. Hababou A. Actualisation de l'apport de l'écho-Doppler couleur dans la caracterisation des lésions mammaires. J Le Sein, 2003; 13(4): 321-323.

24. Levy L. How to describe and classify a breast lesion with ultrasound in 2009? Imagerie de la Femme 2009; 19: 80-99.

25. Raza S, Baum JK. Solid breast lesions: evaluation with power Doppler US. Radiology 1997; 203: 164-168.

26. Santamaria G, Velasco M, Farre X, Vanrell JA, Cardeso A, Fernandez PL. Power Doppler sonography of invasive breast carcinoma: does tumour vascularization contribute to the prediction of axillary status? Radiology 2005; 234 : 374.

27. Byers SW, Sommers CL, Hoxter B, Mercurio AM, Tozeren $A$. Role of $\mathrm{E}$-cadherin in the response of tumor cell aggregates to lymphatic, venous and arterial flow: measurement of cell-cell adhesion strength. J Cell Sci 1995; 108: 2053-2064.

28. Levy L, Michelin J, Teman G, martin B, Dana A, Lacan A, meyer $\mathrm{D}$. Techniques d'exploration radiologique du sein (mammographie, échographie, imagerie par résonance magnétique). Encycl Méd Chir Radiodiagnostic-Principes et téchniques d'imagerie, 34-800-A-10, 2001; 20: 27.

29. Stavros A T, Thickman D, Rapp C L, Dennis M A, Parker $\mathrm{SH}$, Sisney G A. Solid breast nodules: use of sonography to distinguish between benign and malignant lesions. Radiology July 1995 196: 123-134.

30. Buchberger W, Niehoff A, Obrist P, DeKoekkoek-Doll $P$, Dünser M. Clinically and mammographically occult breast lesions: detection and classification with highresolution sonography. Semin Ultrasound CT MR. 2000; 21(4): 325-336.

31. Horvath E, Uchida M, Galleguillos MC, Behnke C, Droguett E, Silva M, et al. Revisión de la Literatura para el Uso del Ultrasonido (US) en Cáncer de Mama - Indicaciones y Requerimientos Mínimos para Asegurar la Calidad del Examen. Medwave 2010; 10(01). 